\title{
Responsabilidad social empresarial en empresas chihuahuenses que obtuvieron el distintivo ESR 2013 ${ }^{1}$
}

\section{Corporate social responsibility in Chihuahuenses companies that obtained the ESR 2013 Award}

Recibido: 17 de agosto de 2015 - Revisado: 19 de septiembre de 2015 - Aceptado: 19 de octubre de 2015

\author{
Alma Lilia Sapién Aguilar ${ }^{2}$ \\ Laura Cristina Piñón Howlet ${ }^{3}$ \\ María del Carmen Gutiérrez Diez ${ }^{4}$
}

\section{Resumen}

La responsabilidad social empresarial(RSE) se ha vuelto una tendencia mundial. El objetivo fue evaluar la implementación de la práctica de responsabilidad social en las empresas chihuahuenses que obtuvieron el distintivo de Empresa Socialmente Responsable (ESR). La naturaleza de la investigación fue mixta, de tipo aplicada y no experimental. La población de interés fueron empresas Chihuahuenses con el distintivo ESR 2013. La mayoría especificó que la principal ventaja de la implementación de prácticas de RSE es la preservación del medioambiente, un mayor rendimiento del capital humano, la atracción de inversionistas y el incremento de las ventas. Además, se puntualizó que se otorga un valor agregado a los servicios dando la oportunidad a las empresas de ser más competitivas y rentables.

\section{Palabras clave}

Responsabilidad social empresarial, implementación, empresas.

\begin{abstract}
Corporate social responsibility (RSE- for its spanish acronym) has become a worldwide trend. Our objective was to evaluate the implementation of the practice of social responsibility in Chihuahuenses companies that obtained the Socially Responsible Company (ESR- for its spanish acronym) award. The nature of the research was mixed, applied and not experimental. The population of interest were Chihuahuenses companies with the ESR 2013 award. Most of them specified that the main advantage of implementing ESR practices is the preservation of the environment, increased performance of human capital, attracting investors and increased sales. Furthermore, it was noted that an added value to services is granted providing an opportunity for companies to be more competitive and profitable.
\end{abstract}

\section{Keywords}

Corporate social responsibility, implementation, companies.
${ }^{1}$ Artículo de investigación. Proyecto de investigación sobre la responsabilidad social en empresas que obtuvieron el distintivo ESR 2013 en la ciudad de Chihuahua, estado de Chihuahua, México.

2 Doctora en Administración de la Universidad Autónoma de Chihuahua (Chihuahua, México). Miembro del Sistema Nacional de Investigadores. Profesora investigadora de la Facultad de Contaduría y Administración de la Universidad Autónoma de Chihuahua.

Correo electrónico:

lsapien@uach.mx

3 Doctora en Administración de la Universidad Autónoma de Chihuahua (Chihuahua, México); Miembro del Sistema Nacional de Investigadores. Profesora investigadora de la Facultad de Contaduría y Administración de la Universidad Autónoma de Chihuahua.

Correo electrónico: lpinon@uach.mx

${ }_{4}$ Doctora en Administración de la Universidad Autónoma de Chihuahua (Chihuahua, México); Profesora investigadora de la Facultad de Contaduría y Administración de la Universidad Autónoma de Chihuahua.

Correo electrónico: cgutierr@uach.mx

Para citar este artículo use: Sapién, A., Piñón, L., \& Gutiérrez, M. (2016) Responsabilidad social empresarial en empresas chihuahuenses que obtuvieron el distintivo ESR 2013. Revista Civilizar Ciencias Sociales y Humanas, 16(30), 223-232. 


\section{Introducción}

La responsabilidad social empresarial (RSE) también conocida como responsabilidad social corporativa representa el compromiso consciente y congruente de cumplir integralmente con la finalidad de la empresa, tanto en lo interno como en lo externo. Este compromiso considera las expectativas económicas, sociales y ambientales de todos sus participantes. Al mismo tiempo, se demuestra respeto por la gente, los valores éticos, la comunidad y el medioambiente (Swarup, 2005), contribuyendo así a la construcción del bien común (Cajiga, 2011). Carroll (1991) sugirió que el concepto de RSE debería suponer las siguientes cuatro dimensiones: económica, legal, ética y filantrópica.

Para este autor cada dimensión debe tomar en cuenta el vínculo con los usuarios, propietarios, clientes, empleados, comunidad y público en general. La RSE tuvo su origen en los Estados Unidos de Norteamérica, enfocándose en su inicio, en una visión paternalista de las relaciones sociedad/empresa. Esto se debió a que el sistema capitalista, deseaba competir o no quedarse al margen del socialismo en el esquema de interés público. Así mismo, se fortaleció el pensamiento crítico sobre el papel de las empresas en el crecimiento económico y los abusos hacia la sociedad.

En consecuencia, los empresarios se vieron obligados a adoptar comportamientos para proteger a los consumidores, el medioambiente, la salud de sus colaboradores y los derechos de las minorías (Debra \& McKinnon 2001; Escobar, 2012). Esta filosofía se propagó por Europa en la década de los ochenta del siglo pasado.

La primera organización mexicana en este esquema se llamó Alianza por la Responsabilidad Social Empresarial. Esta estructura agrupaba alrededor de 19 organizaciones con importantes antecedentes de trabajo. El propósito era incentivar a que las empresas fueran y se percibieran como creadoras de valor y generadoras de bien común. Todo esto a través del ejercicio de su responsabilidad social, apalancando, coordinando y facilitando la sinergia de los esfuerzos de las organizaciones en beneficio del país y, en particular, de todos los participantes (Cajiga, 2011).

Es menester señalar que una característica del distintivo ESR es que no es una certificación oficial, ya que la única autoridad en México para certificar es el Gobierno. El distintivo ESR debe considerarse un instrumento de autoevaluación, que es conducido por una asociación sin fines de lucro y que propone relaciones entre Gobierno y organizaciones civiles (Guerra, 2011).

Según el Centro Mexicano para la Filantropía (2013) Chihuahua cuenta con 17 empresas con el distintivo ESR, que sin duda agrega valor a la marca y ofrece mayor rentabilidad. Al mismo tiempo, acredita a las firmas ante sus empleados, inversionistas, clientes, autoridades y sociedad en general, por su trabajo constante y la anexión institucional a políticas de preservación del medioambiente, desarrollo social y, por supuesto, a estrategias para el crecimiento profesional y humano de su planta laboral. Al considerar todos estos antecedentes, el objetivo de este estudio fue analizar las acciones de RSE que están llevando a cabo las empresas chihuahuenses que cuentan con el distintivo ESR 2013. Estos resultados serán de gran importancia para evaluar su impacto social, ventajas y desventajas, y para sopesar si otras empresas similares merecen replicar estas buenas prácticas e invertir en estas acciones sociales.

\section{Materiales y métodos}

La naturaleza de la investigación fue mixta, de tipo básica, no experimental y según un diseño transaccional descriptivo. La propuesta en su etapa operativa se apegó al método induc- 
tivo-documental. El trabajo de investigación se realizó en la ciudad de Chihuahua, México, de febrero de 2013 a abril de 2014. La población fue la responsable del área de RSE de las empresas chihuahuenses que obtuvieron el distintivo ESR por parte del Centro Mexicano para la Filantropía en el 2013.

Se analizó en gabinete la base de datos del Centro Mexicano para la Filantropía, donde se encontraron las 17 compañías que obtuvieron el distintivo ESR 2013. Las empresas participantes en el estudio fueron: Casa Myers, Pavos Parson, Aceites Lubricantes de Chihuahua, Alsuper, Aurico Gold de México, Grupo Catraar México, Abitat, Intermex Parques Industriales, S-Mart, Compañía Minera Dolores, Coeur Mexicana, El Heraldo de Chihuahua, Instituto Tecnológico y de Estudios Superiores de Monterrey, Campus Chihuahua; Agnico Eagle México, American Industries Group, Grupo Cemento de Chihuahua e Interceramic.

Los indicadores que describieron a la variable de RSE fueron "vinculación con la comunidad", "vinculación con el medioambiente", "ética" y "gobernabilidad empresarial". La herramienta para la recolección de datos fue un cuestionario especialmente diseñado para alcanzar los objetivos planteados. Este se dividió en dos apartados: el primero para obtener datos generales (algunos opcionales para guardar la privacidad de las empresas) y el segundo para cuantificar las actividades de ESR. En este segundo apartado se aplicaron dos preguntas abiertas, tres de opción múltiple y una de matriz de múltiples respuestas numéricas.

Cada ítem se valoró con una escala de uno a cinco (1 a 5): 12 ítems de ética y Gobierno corporativo, 10 de vinculación con el medioambiente, 13 de calidad de vida en la empresa y 14 de vinculación con la comunidad. Se solicitaron también las respuestas en preguntas abiertas, y se les especificó a los participantes que señalaran cuáles consideraban sus áreas de oportunidad en cuanto a RS, a fin de emitir recomendaciones. La información se codificó en el programa en línea SurveyMonkey y su análisis e interpretación fueron de tipo estadísticodescriptivo.

\section{Resultados}

Del total de las compañías encuestadas, el $88 \%$ son grandes empresas (con más de cien colaboradores) y el $12 \%$ restante medianas empresas. El $24 \%$ de las empresas con distintivo ESR pertenece al sector minero y extractivo, el $12 \%$ a la industria manufacturera, el $18 \%$ a la construcción y al comercio, el $6 \%$ a empresas de servicios y el $40 \%$ a otros sectores donde destacan la industria de la cerámica, la educación y el área de distribución.

La mayoría de las empresas encuestadas cuenta con políticas de respeto a la competencia y contempla políticas anticorrupción. La publicidad que maneja en los productos o servicios se basa en la realidad y, además, esta mayoría tiene mecanismos de comunicación entre los clientes y módulos para que se hagan sugerencias, quejas o reclamos. Un porcentaje alto tiene programas de apoyo y desarrollo de proveedores locales e implementa políticas de honradez y atención al cliente. Una gran parte presenta algún sistema de monitoreo de la actuación ética, un $90 \%$ emite reportes de RSE y otro porcentaje igual cuenta con políticas de equidad en selección y pago a proveedores. Alrededor de $86 \%$ de las empresas cumple a tiempo con las obligaciones fiscales y atiende las solicitudes de Gobierno. 
Gráfica 1.

Ética y gobernabilidad empresarial

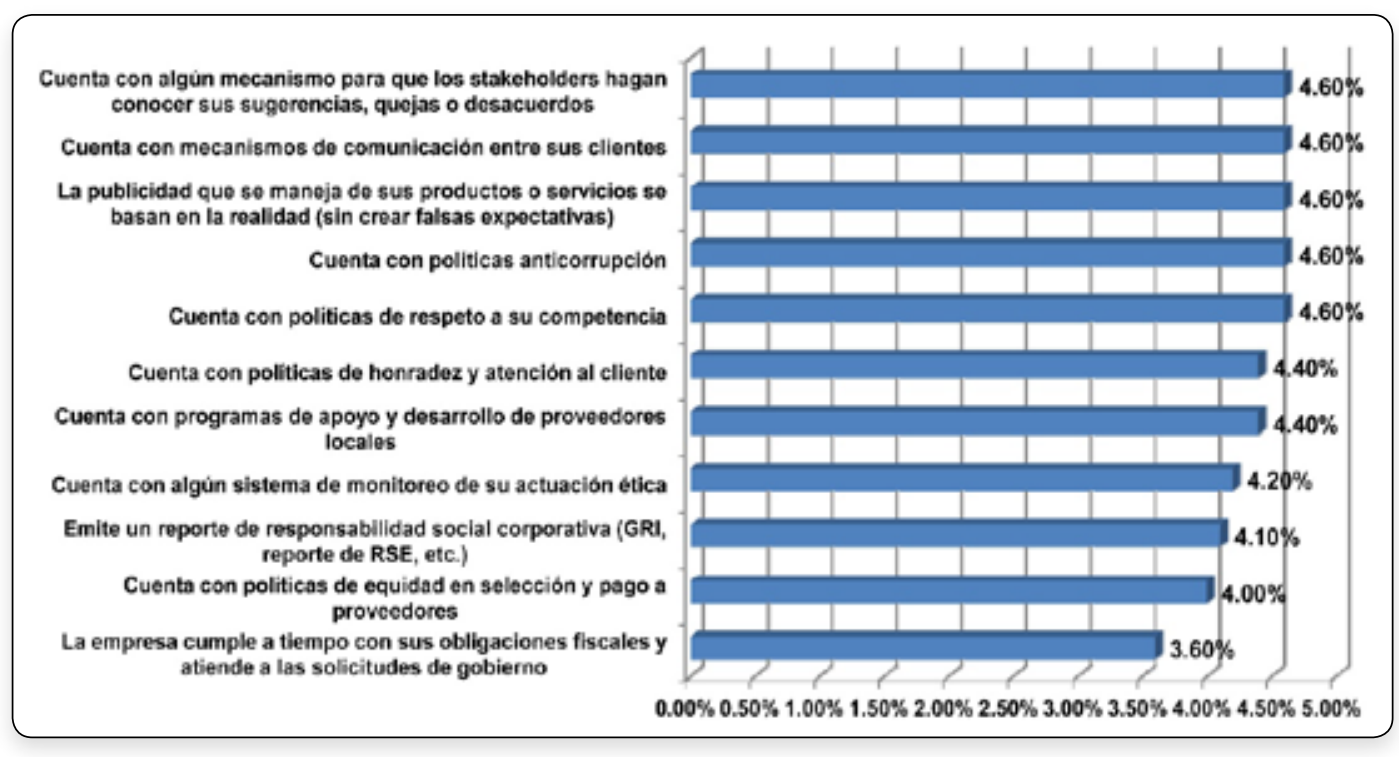

Fuente: Sapién, 2014.

La mayoría de las empresas procura reciclar o reutilizar los activos fijos y productos no utilitarios, mientras que otro gran porcentaje emplea la materia prima e insumos principalmente reciclables. Un $90 \%$ de las empresas cuenta con algún método de medición para el aprovechamiento de recursos, mientras que $89 \%$ aplica alguna política de cuidado del medioambiente. Un $89 \%$ de las empresas involucra a los cola- boradores en la concientización y programas medioambientales, al igual que destina alguna partida a programas de conservación ambiental. $75 \%$ de las firmas selecciona a proveedores que promueven las buenas prácticas del cuidado ambiental, un $74 \%$ posee sistemas o apartados para disminuir su huella de carbono y un $60 \%$ cuenta con políticas de reducción de consumo de energía eléctrica, agua y productos tóxicos.

Gráfica 2.

Vinculación con el medioambiente

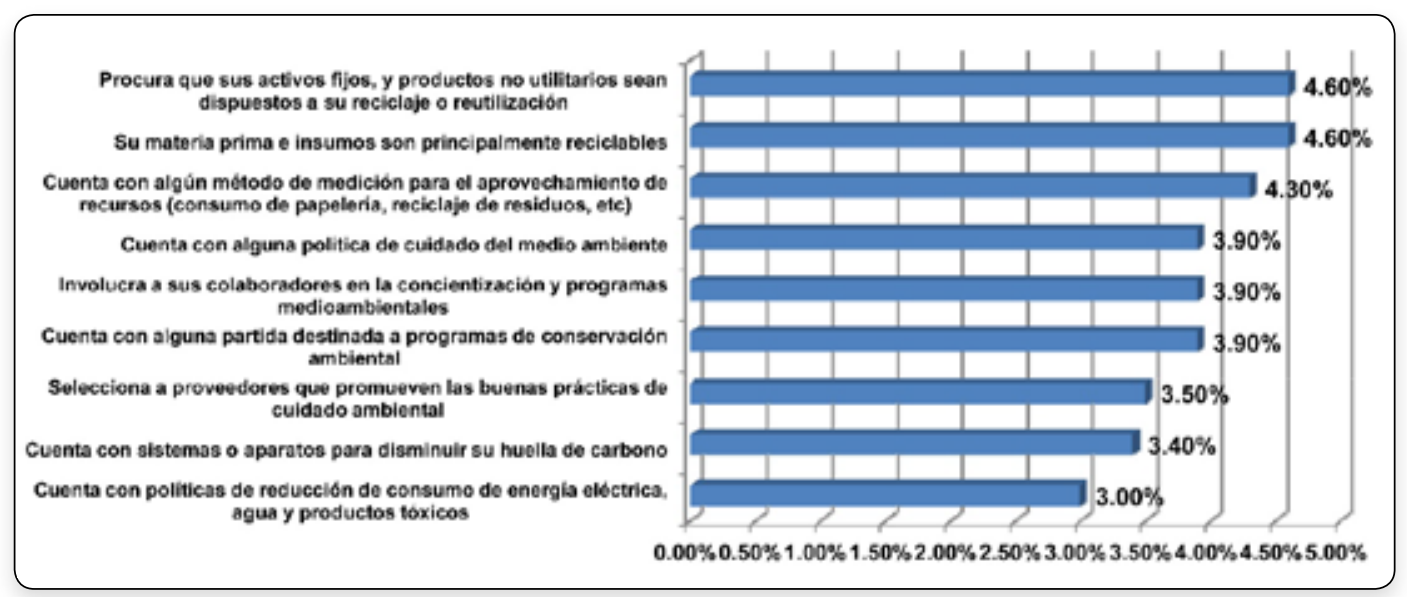

Fuente: Sapién, 2014. 
Los resultados mostraron que todas las empresas encuestadas, fomentan el trabajo en equipo y valoran las opiniones de los empleados para la toma de decisiones. La gran mayoría cuenta con código de ética y conducta mientras que un $96 \%$ de las compañías tiene políticas y procedimientos alineados con el código de ética, así como con instrumentos de comunicación entre los colaboradores para la toma de decisiones y sugerencias. Un $96 \%$ de las firmas maneja esquemas de trabajo flexible que se acomoden a las necesidades del personal, mientras que un $94 \%$ implementa políticas de no discriminación.
E1 $92 \%$ participa con apoyos educativos y capacitación a los trabajadores para el desarrollo de habilidades, además de aplicar prácticas laborales que promueven el equilibrio entre el trabajo y la familia. Cerca de un $96 \%$ cuenta con políticas de liquidaciones y despidos honestos y $90 \%$ tiene programas de estímulos al personal por su participación. Un $88 \%$ otorga prestaciones a los colaboradores que son superiores a las de la ley así como $86 \%$ tiene políticas de equidad de género. El $78 \%$ de las empresas implementa programas de seguridad y salud al personal en el área de trabajo.

Gráfica 3.

\section{Calidad de vida en la empresa}

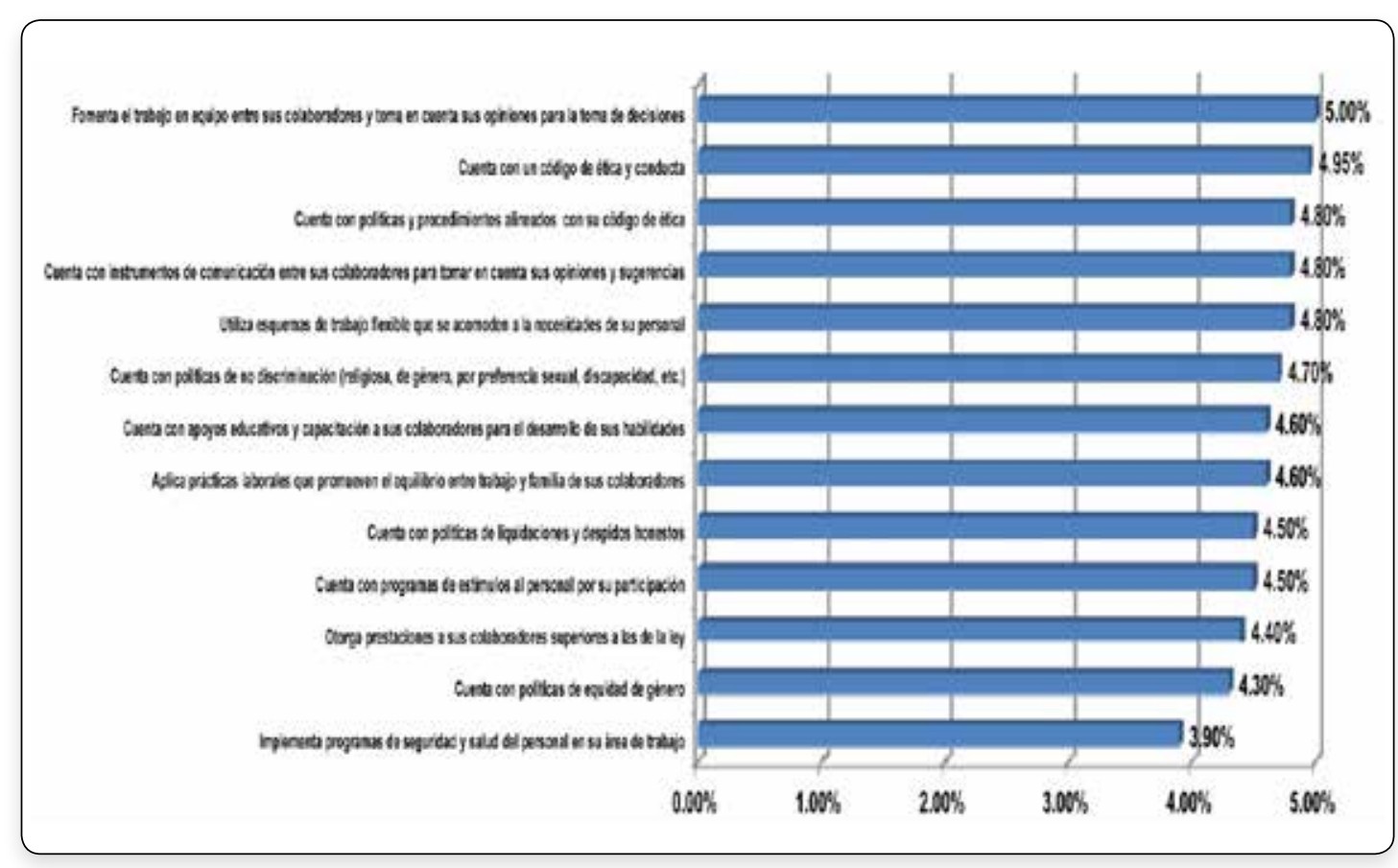

Fuente: Sapién, 2014.

En la vinculación con la comunidad, un $98 \%$ de las empresas mantiene alianzas con organizaciones a beneficio de la comunidad. La mayoría de las compañías promueve el bienestar económico y social de la comunidad en la que opera, otorga donativos en efectivo a asociaciones civiles con causa social y participa con apoyos no financieros en diversas actividades de la comunidad.
Cerca del $92 \%$ de las firmas presenta mecanismos de comunicación entre la comunidad en la que opera e identifica sus necesidades. Alrededor de un $92 \%$ de las empresas tiene una fundación empresarial, mientras que el $90 \%$ promueve la filantropía entre los clientes y usuarios. Cerca del $88 \%$ tiene políticas de apoyo social y constata un mecanismo de medición del impacto en la comunidad, el $82 \%$ 
realiza actividades sociales o comunitarias que fomenten los valores, promueve el voluntariado corporativo y establece mecanismos de merca- dotecnia alineados con el beneficio social. E1 70 $\%$ impulsa el desarrollo de la comunidad en la que se inserta.

Gráfica 4.

Vinculación con la comunidad

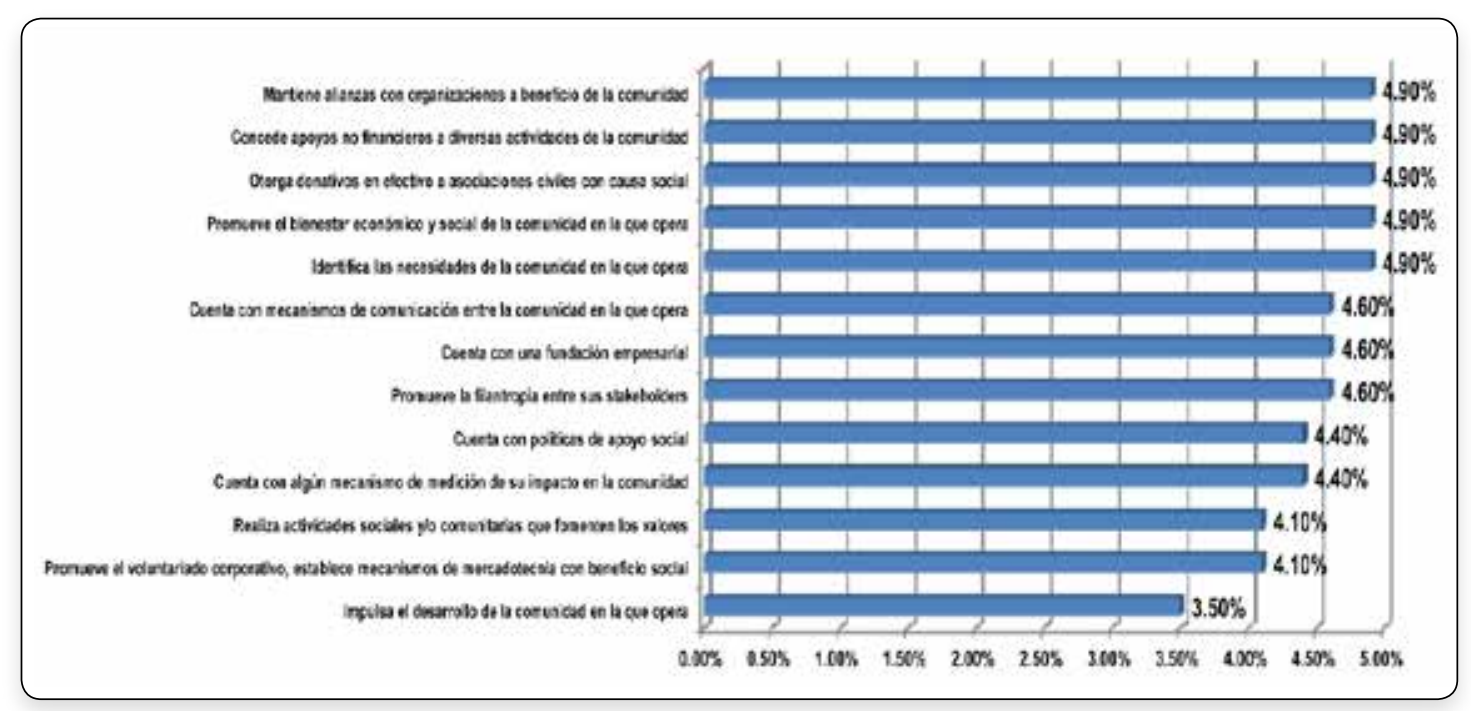

Fuente: Sapién, 2014.

En cuanto a las acciones de RS, en el apartado sobre la calidad de vida en la empresa, nueve de las empresas contestaron que sus acciones son capacitaciones que se ejecutan en el departamento de vinculación con el medioambiente y seis que sus acciones son donativos de árboles y cabinas de reciclajes. Con respecto a ética y gobernabilidad empresarial, dos empresas señalaron que las acciones en este apartado son el control de activos y tener políticas de prevención de pérdidas y un Gobierno cooperativo.

En la evaluación del departamento de vinculación con la comunidad, no se obtuvo respuesta por parte de las empresas. Ocho de las compañías destacaron que la mayor ventaja de la implementación de la filosofía de RSE es la protección al medioambiente. Seis enfatizaron un más alto rendimiento del capital humano, la atracción de inversionistas, el incremento de ventas y el valor agregado. Cuatro se refirieron al mejoramiento de políticas y control interno y, por último, dos firmas reportaron el aumento de la competitividad y rentabilidad.

Las empresas realizan otras acciones de RSE en el ámbito de ética y gobernabilidad empresarial tales como el establecimiento de políticas de Gobierno corporativo, control de activos, prevención de pérdidas y políticas de despido. Así mismo, cuentan con comités de ética y programas de denuncia de casos no éticos. Por otro lado, tienen participaciones en la Bolsa Mexicana de Valores.

Respecto al ámbito de vinculación con el medioambiente, ejecutan actividades como la donación de árboles y cabinas de reciclaje, así como contar con la norma ISO 26000. Otras acciones en el ámbito de calidad de vida que llevan a cabo son capacitaciones, apoyo en becas educativas para los colaboradores y las familias y el contar con el distintivo "Great Place to Work". En el ámbito de vinculación con la comunidad, cuentan con el sistema de redondeo en tiendas y la realización de carreras empresariales. 


\section{Discusión}

Esta investigación pone de manifiesto que las empresas chihuahuenses con el distintivo ESR 2013 practican los lineamientos de RSE en las áreas de ética y gobernabilidad empresarial, calidad de vida en la empresa; vinculación con la comunidad y vinculación con el medioambiente con el propósito de garantizar su crecimiento y permanencia en el mercado, así como también ser amables con el medioambiente. A este respecto Blank (2002) indica que todas las organizaciones buscan crecer, con el fin de obtener un beneficio que les permita ser sostenibles, crear una imagen corporativa positiva, implementar el bienestar institucional y garantizar su permanencia y consolidación en el ámbito empresarial; la RSE puede contribuir con este propósito.

La mayoría de las empresas señala que la principal ventaja de la implementación de prácticas de RSE es la preservación del medioambiente, seguida (en grado de importancia) de un mayor rendimiento del capital humano, la atracción de inversionistas a las empresas, el incremento de las ventas (lo que otorga un valor agregado a los servicios y productos), mejoramiento de las políticas de control interno, aumento de la competitividad y la rentabilidad; coadyuvando todo esto a reforzar los valores de la empresa.

Bigne y Currás (2008) sostienen que la RSE es un fenómeno que ha ayudado a que cada vez más los consumidores introduzcan criterios sociales y medioambientales en sus decisiones de consumo; lo cual es congruente con los resultados obtenidos en esta investigación. A este respecto Rochlin (2005) resalta que cada vez son más las firmas que se están comprometiendo con la RSE y están conscientes de que sus acciones y operaciones tienen un profundo impacto en la sociedad que les rodea, no tratando de maximizar las utilidades si estas se generan con malas prácticas laborales como la degradación del medioambiente o el uso de prácticas éticas cuestionables; concordando esta aseveración con los resultados expuestos.

Vives (2012) enuncia: son muchos los que alegan que la RSE no rinde beneficios o que es un gasto inútil, porque no ven el nexo entre esas actividades y la mejora en los rendimientos. Algunos llegan a atacar las prácticas responsables citando ejemplos de empresas supuestamente responsables que tienen problemas de rendimiento financiero o de aceptación en el mercado. Esto se opone con lo que las empresas socialmente responsables señalaron, ya que para ellas los beneficios superan los costos en este rubro.

Para Verduzco (2006) la perspectiva más difundida internacionalmente es la que identifica la RSE con el desempeño corporativo, entendiendo esto como el papel que deben ejercer las empresas en beneficio de sus respectivas comunidades. En este sentido, las empresas socialmente responsables sujetos de esta investigación, son conscientes del creciente desafío de cuantificar el impacto que sus actividades, procesos, productos y servicios tienen sobre el medioambiente y el entorno social, enfrentando la tarea de ser capaces de alinear su quehacer con los retos que surgen de un compromiso de responsabilidad social.

\section{Conclusiones}

La investigación reveló que el capacitar en forma permanente al personal e implementar programas de seguridad y salud del personal en las áreas de trabajo son parte del éxito de la responsabilidad social. Resulta claro que es necesario impulsar el desarrollo en las comunidades en las que se opera y coordinar acciones en conjunto con las organizaciones de la sociedad civil.

Se hace ineludible cumplir a tiempo con las obligaciones fiscales y atender a las solicitudes del Gobierno, así como emitir reportes de RSE y unirse a los estándares del Global Reporting Initiative. Algunas empresas expre- 
saron como limitantes la carencia de presupuesto y de personal que coordine las actividades, el rechazo de los colaboradores frente al cambio, la falta de involucramiento de personal de la empresa, la escasez de tiempo, la apatía por parte de los grupos de interés, el alto costo de la obtención del distintivo y la ausencia de apoyo del Gobierno estatal.

La RSE se ha vuelto una tendencia mundial, no solo porque brinda una estrategia de mercadotecnia, sino porque garantiza el crecimiento y permanencia de las organizaciones a través de los diversos lineamientos que se han desarrollado a partir de la réplica de diversas prácticas, así como la aplicación de estándares de sustentabilidad. Como estrategias adicionales para la implementación de prácticas de RSE, se sugiere reforzar las acciones de vinculación con el medioambiente mediante la norma ISO 26000, la cual está diseñada para ser utilizada por organizaciones de todo tipo, tanto en los sectores públicos como privados, en los países desarrollados y en vía de desarrollo, así como en las economías en transición. La norma les ayudará en su esfuerzo por operar de la manera socialmente responsable que la sociedad exige cada vez más.

\section{Recomendaciones}

Introducir cambios en los procesos y prácticas para mejorar los impactos económicos, sociales y ambientales, construyendo un sistema de gestión para asegurar la permanencia de los cambios inducidos por la RSE.

Practicar el "decálogo" de responsabilidad social del Centro Mexicano para la Filantropía (Porto \& Castromán, 2006) y el proceso de la senda Kyosei (Kaku, 2005). Donde se expresa que la cooperación de todos los empleados es esencial para lograr un equilibrio entre lo económico, social y ambiental.

Establecer una partida de dinero para tareas de responsabilidad social en las empresas, teniendo en cuenta este apartado en la planeación estratégica de las organizaciones. Es necesario examinar las acciones de desempeño social para evaluar si se está cumpliendo con los objetivos de empresas socialmente responsables.

\section{Referencias}

Bigne, E., \& Currás, R. (2008). ¿Influye la imagen de responsabilidad social en la intención de compra? El papel de la identificación del consumidor con la empresa. Universia Business Review, 19, 10-23. Recuperado de http://www. redalyc.org/pdf/433/43301901.pdf

Blank, B. L. (2002). La Administración de Organizaciones: Un Enfoque Estratégico (3a ed.). Cali: Artes Gráficas del Valle.

Cajiga,F.(2011). El concepto de responsabilidad social empresarial. México: Centro Mexicano para la Filantropía Recuperado de http://www.cemefi.org/esr/images/ stories/pdf/esr/concepto_esr.pdf

Carroll, A. B. (1991). The pyramid of Corporate Social Responsibility: Toward the moral management of organizational stakeholders. Business Horizons, 34(4), 39-48.

Centro Mexicano para la Filantropía, A.C. (2013). Cuestionario ESR 2013 Empresas Grandes. Distrito Federal, México: Autor.

Debra, K., \& McKinnon, A. (2001). Who cares? How to encourage corporations to adopt socially responsible corporate citizenship practices. Australia: The Journal of Corporate Citizenship. Greenleag Publishing. Recuperado de http://www.highbeam. com/doc/1G1-79338841.html

Escobar, M. (2012). Asociación Colombiana de Facultades de Administración. Recuperado de http://aprendeenlinea.udea.edu.co/ $1 \mathrm{~ms} /$ moodle/file.php/805/Ponencias_I_ 
Seminario_Internacional_y_II_Nacional_de_Familias_ContemporA_neas_y_ PolA_ticas_PAoblicas.pdf

Guerra, E. (2011). ¿Para qué sirve el Distintivo ESR...?. Expoknews. Recuperado de http://www.expoknews.com/para-quesirve-el-distintivo-esr/

Kaku, R. (2005). La senda Kyosei. Harvard Business Review, 83(8), 105-113.

Porto, N., \& Castromán, J. (2006). Responsabilidad social: un análisis de la situación actual en México y España. Contaduría y Administración, 220, 67-88.

Rochlin, S. (2005). Llevar la responsabilidad corporativa al ADN de su empresa. Harvard Business Review, 83(8), 31-38.
Swarup, K. M. (2005). Corporate Social Responsibility. Present practices and future possibilities. Indian Journal of Industrial Relations, 40(4), 547-557.

Verduzco, A. (2006). Responsabilidad social empresarial: de la dimensión corporativa a la personal. The Anáhuac Journal, 6(1), $100-111$.

Vives, A. (2012). Sustainability indices in emerging markets: Impact on responsible practices and financial market development. Journal of Sustainable Finance and Investment, 2(3-4), 318-337. 
